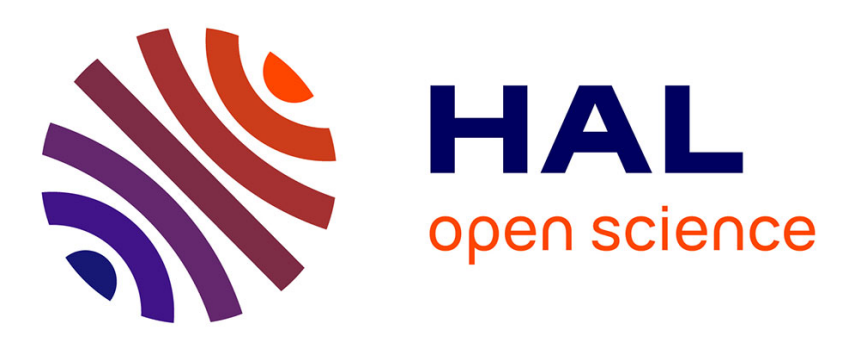

\title{
Robust quantum coherence above the Fermi sea
}

\author{
S. Tewari, P. Roulleau, C. Grenier, F. Portier, A. Cavanna, U. Gennser, D. \\ Mailly, P. Roche
}

\section{To cite this version:}

S. Tewari, P. Roulleau, C. Grenier, F. Portier, A. Cavanna, et al.. Robust quantum coherence above the Fermi sea. Physical Review B: Condensed Matter and Materials Physics (1998-2015), 2016, 93 (3), pp.035420. 10.1103/PhysRevB.93.035420 . cea-01366658

\section{HAL Id: cea-01366658 https://hal-cea.archives-ouvertes.fr/cea-01366658}

Submitted on 15 Sep 2016

HAL is a multi-disciplinary open access archive for the deposit and dissemination of scientific research documents, whether they are published or not. The documents may come from teaching and research institutions in France or abroad, or from public or private research centers.
L'archive ouverte pluridisciplinaire HAL, est destinée au dépôt et à la diffusion de documents scientifiques de niveau recherche, publiés ou non, émanant des établissements d'enseignement et de recherche français ou étrangers, des laboratoires publics ou privés. 


\title{
Robust quantum coherence above the Fermi sea
}

\author{
S. Tewari, ${ }^{1,2}$ P. Roulleau, ${ }^{1}$ C. Grenier, ${ }^{3}$ F. Portier, ${ }^{1}$ A. Cavanna, ${ }^{2}$ U. Gennser, ${ }^{2}$ D. Mailly, ${ }^{2}$ and P. Roche ${ }^{1, *}$ \\ ${ }^{1}$ Nanoelectronic Group, SPEC, CEA, CNRS, Université Paris-Saclay, F-91191 Gif-sur-Yvette, France \\ ${ }^{2}$ Phynano Team, Laboratoire de Photonique et Nanostructures, CNRS, Route de Nozay, F-91460 Marcoussis, France \\ ${ }^{3}$ Institute for Quantum Electronics, ETH Zurich, CH-8093 Zurich, Switzerland
}

(Received 28 January 2015; revised manuscript received 18 November 2015; published 14 January 2016)

\begin{abstract}
In this paper we present an experiment where we measured the quantum coherence of a quasiparticle injected at a well-defined energy above the Fermi sea into the edge states of the integer quantum Hall regime. Electrons are introduced in an electronic Mach-Zehnder interferometer after passing through a quantum dot that plays the role of an energy filter. Measurements show that above a threshold injection energy, the visibility of the quantum interferences is almost independent of the energy. This is true even for high energies, up to $130 \mu \mathrm{eV}$, well above the thermal energy of the measured sample. This result is in contradiction with our theoretical predictions, which instead predict a continuous decrease of the interference visibility with increasing energy.
\end{abstract}

DOI: 10.1103/PhysRevB.93.035420

A new type of quantum device, relying on the onedimensional edge states of the quantum Hall regime, where electrons mimic the photon trajectory of a laser beam, has opened a route towards electron quantum optics and the manipulation of single electron excitations [1-3]. Pauli statistics and interactions provide new ingredients for the physics of the electrons which are not relevant for photons. For example, when electrons are injected above the Fermi sea, it is fundamental to understand how their phase coherence will be affected by the injection energy. We explore this issue by first using a quantum dot to inject the carriers at a controllable energy into an edge state. Then an electronic Mach-Zehnder interferometer is used to monitor the quantum coherence of the electronic quasiparticle. We find that above a certain threshold, the coherence is energy independent; it is even preserved at energies 50 times larger than the electronic temperature. This is remarkable, since from simple considerations based on Fermi's golden rule, one would expect that the relaxation rate increases with the injection energy, thus reducing quantum coherence. Indeed, our simulations using recent theories [4] predict a continuous trend of increasing relaxation. While the origin of this coherence robustness remains unidentified, it has a significant bearing for the implementation of quantum information encoded in electron trajectories [5-8].

The edge states used in this new type of device for electronic quantum optics are obtained by applying a high magnetic field perpendicular to a high mobility two-dimensional (2D) electron gas. When the number of electrons per quantum of flux (the filling factor) is an integer, the transport occurs through one-dimensional channels located at the edge of the sample. The electron motion in these wires is chiral: The electrons drift in one direction with a speed of the order of $10^{4}-10^{6} \mathrm{~ms}^{-1}[9,10]$, thus compensating the confining electric field with the Lorentz force. Much recent progress has been made in the understanding of decoherence mechanisms and the energy exchanges in the integer quantum Hall regime at a filling factor of 2 . In this regime, there are two parallel, adjacent channels at each edge in the sample. The Coulomb interaction has been shown to play a key role in these systems:

*patrice.roche@cea.fr (i) The quantum coherence at finite temperature is limited by the thermal charge noise of the environment [11,12]; (ii) there is an energy relaxation in out-of-equilibrium edge states and an energy transfer between the two edge states [13]; and (iii) the visibility of quantum interferences in electronic Mach-Zehnder interferometers exhibits a side lobe structure at finite bias, which is explained by a beating effect between a neutral and a charged excitation shared by the two edge states [4,14-17]. Here, we inject a quasiparticle at a well-defined energy above the Fermi sea into an edge channel. We then explore to which extent such a single charge behaves as a free noninteracting particle in this interacting quantum system, thus directly probing the validity of the Landau's Fermi liquid picture $[18,19]$. While extensively studied in diffusive quantum conductors, this question has yet to be addressed experimentally for the case of one-dimensional chiral conductors. We note, however, that this is a key point for quantum information experiments using electrons transported through edge channels. Experimentally, we use a quantum dot (QD) as an energy filter, and a Mach-Zehnder interferometer to probe the injected quasiparticle's loss of phase coherence.

The QD consists of two quantum point contacts (QPCs) and a plunger gate (see Fig. 1). Figure 2(a) shows a 2D plot of the transmission probability through the dot as a function of the drain-source bias and the plunger gate voltage $V_{P}$. The transmission probability is defined as $d I_{T} / d I_{0}$, where $I_{T}$ is the transmitted current through the QD and $I_{0}$ is the impinging current. From this measurement we deduce the lever arm of the plunger gate $\alpha=d \epsilon / d\left(e V_{P}\right)=1.46 \times 10^{-4}$, where $\epsilon$ is the QD energy. As an example, Fig. 3 (top graph) displays the current $I_{T}$ as a function of $V_{P}$. It is clear that $I_{T}$ is almost constant for energies varying between 0 and $\mathrm{e} V_{\mathrm{DS}}$. This signals an absence of excited states for this particular tuning of the quantum dot. We deduced an electronic temperature of $31 \pm 1 \mathrm{mK}$ by fitting the resonance of the transmission probability [see Fig. 2(b)] as a function of $V_{P}$ with $d I_{T} / d I_{0} \propto$ $\cosh ^{-2}\left(\delta / 2 k_{B} T\right)$ [20], where $\delta=\alpha\left(e V_{P}-e V_{P_{0}}\right)$ is the energy difference between the QD energy level and the Fermi level, and $V_{P_{0}}$ is the plunger gate voltage which maximizes the conductance. Indeed, the intrinsic width of the energy filter is much smaller than the electronic temperature. In practice, the presence of excited states in the dot limited the energy 


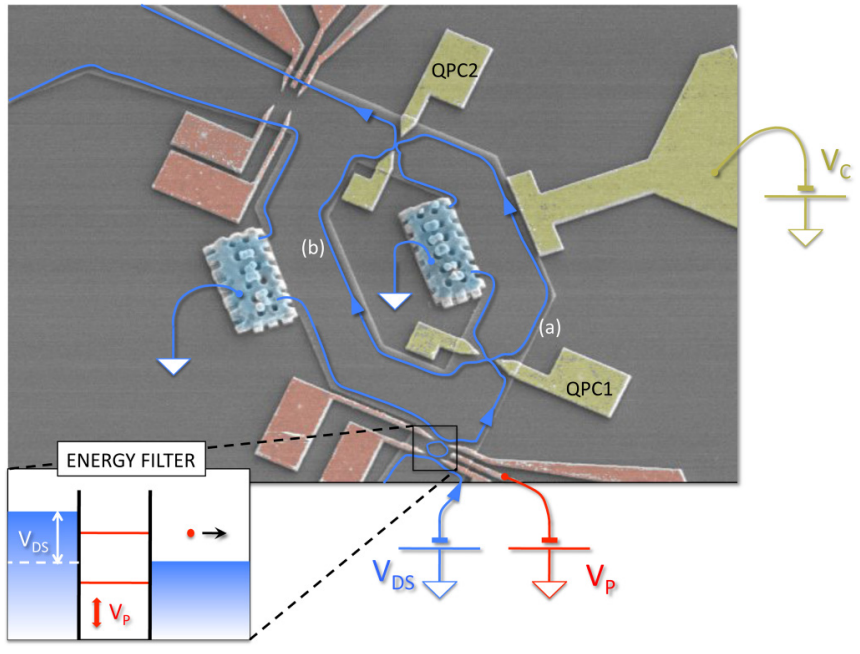

FIG. 1. Measured device. Colorized scanning electron microscope view of the sample before the final fabrication step in which the gates and ohmic contacts are connected to larger connecting pads. The sample consists of a quantum dot in series with an electronic Mach-Zehnder interferometer (MZI). The quantum point contacts (QPCs) and gates controlling the dot are in red while those controlling the MZI are in yellow. QPC1 and QPC2 serve as the two beam splitters of the MZI. Note the small ohmic contacts in blue connected to the ground, which prevents spurious quantum interferences inside the interferometer. The chiral trajectory of the excitations in the outer edge state is schematically represented in blue, and the quantum interference takes place between the trajectories (a) and (b). The mesa is $1.2 \mu \mathrm{m}$ wide, each arm of the Mach-Zehnder interferometer is $7.2 \mu \mathrm{m}$ long, and the distance between the dot (the energy filter) and the MZI is $2.8 \mu \mathrm{m} . V_{P}$ is the potential applied on the plunger gate of the dot, allowing a control of the relative position of the energy levels of the dot compared to the Fermi level. The quantum interferences in the MZI are revealed by sweeping the gate voltage $V_{C}$, which modifies the trajectory length difference between (a) and (b).

range we explored to $120 \mu \mathrm{eV}$, close to the addition energy estimated to be of the order of $150 \mu \mathrm{eV}$ [see the Supplemental Material (SM) [21]].

Once the QD is characterized, we measure the current through the whole system, constituted by the QD in series with the MZI, and operated in its optimal parameter regime for maximum visibility (see SM [21]). Probing the decoherence at finite energy is simply realized by measuring the relative amplitude of the current oscillations revealed by sweeping the central gate voltage $V_{C}$ of the Mach-Zehnder interferometer. A color plot of the current as a function of $V_{C}$ and $V_{P}$ is displayed in Fig. 3. This plot corresponds to experiments with five different drain-source voltages $V_{\mathrm{DS}}$. One would expect a current injection only for a range of $V_{P}$ values between 0 and $V_{\mathrm{DS}}$ (see the inset of Fig. 1), and this is indeed what can be seen in the top graph of Fig. 3, where the window of nonzero current (around $25 \mathrm{pA}$ ) increases linearly with increasing source-drain voltage. Sweeping $V_{C}$ while $V_{P}$ is within this window reveals the quantum interferences. The interference pattern is clearly observable in Fig. 3. It is easily noticed that, within the window for nonzero current, the amplitude
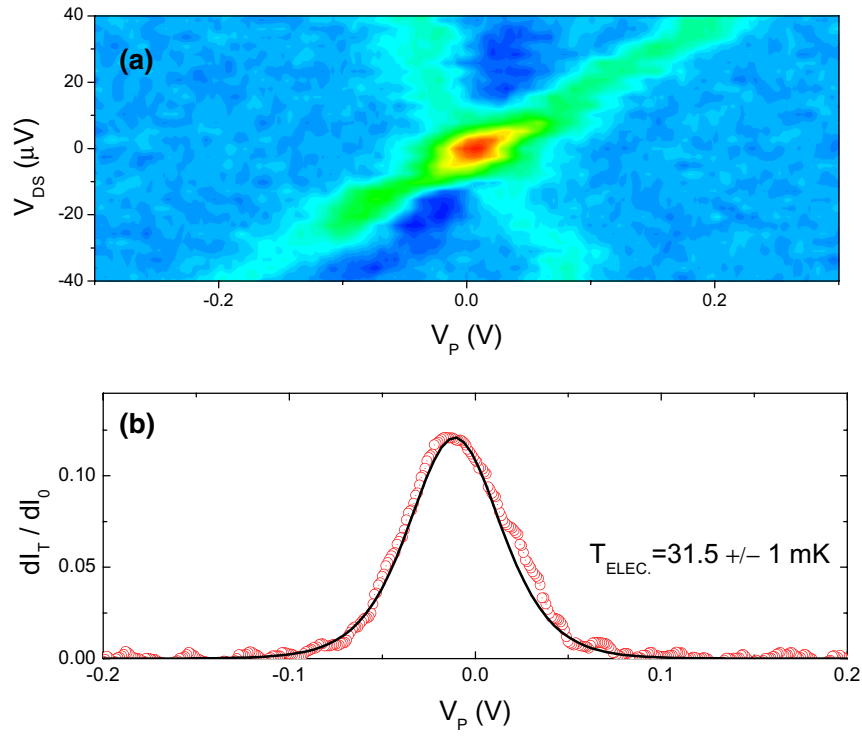

FIG. 2. (a) Color plot of the transmission probability through the quantum dot as a function of the plunger voltage $V_{P}$ and the drain source voltage $V_{\mathrm{DS}}$. (b) Transmission probability as a function of $V_{P}$ at zero bias. The continuous line is a fit of this resonance.

of the current oscillations appears to change very little, a clear indication that the quantum interferences, and hence the coherence, are more or less independent of the injection energy of the electrons.

To be more quantitative, we plot in Fig. 4 the visibility $\frac{I_{\max }-I_{\min }}{I_{\max }+I_{\min }}$ of quantum interferences as a function of the injected energy. Surprisingly, for energies greater than $20 \mu \mathrm{eV}$, the visibility remains almost constant instead of decreasing down to zero as expected. We need to consider whether the saturation of the visibility is due to an unexpected robustness of the

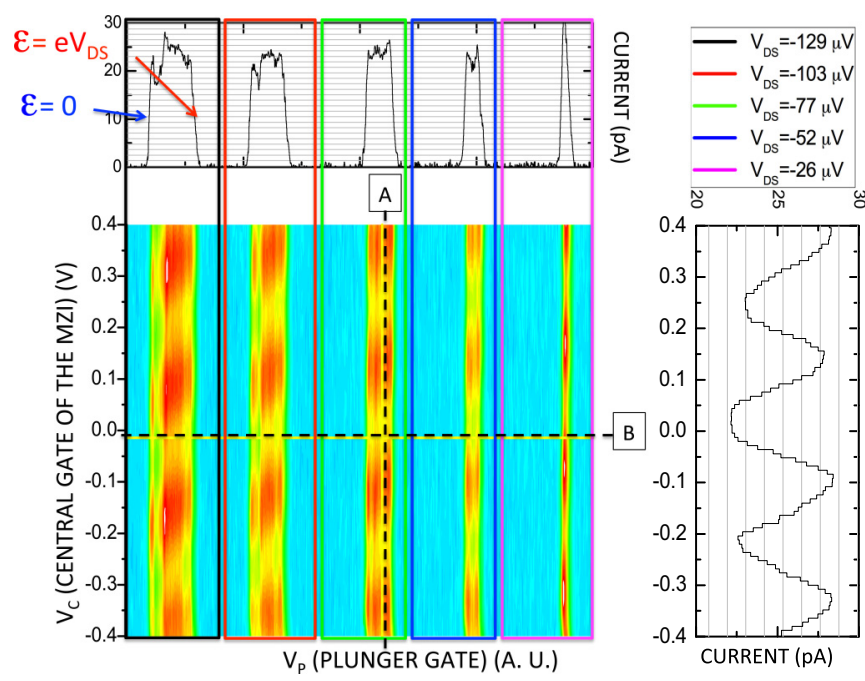

FIG. 3. Current as a function of the plunger gate voltage $V_{P}$ and the central gate voltage $V_{C}$ of the MZI for different values of the drain source bias $V_{\mathrm{DS}}$. The top graph, representing the current as a function of $V_{P}$, corresponding to a $2 \mathrm{D}$ plot cut through line $\mathrm{B}$. The right graph shows the interference pattern as a function of $V_{C}$ corresponding to a cut through line A. 


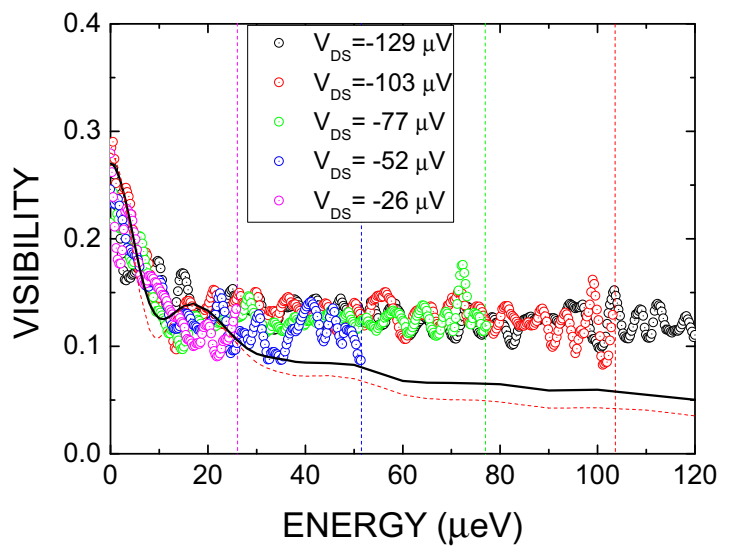

FIG. 4. Visibility of quantum interferences as a function of the energy for five different bias voltages. The vertical lines represent the maximum energy achievable for each bias. The solid and the dashed lines show the calculated visibility for interaction parameters $\theta=\pi / 2$ and $\theta=\pi / 3$, respectively.

quantum coherence, or e.g., to the finite distance, $2.7 \mu \mathrm{m}$, between the energy filter and the MZI. Indeed, a short relaxation length $(\ll 2.7 \mu \mathrm{m})$ would mean that the electrons injected above the Fermi sea are fully relaxed before reaching the entrance of the interferometer. However, this scenario is in contradiction both with recent experimental results and theories, as we will now show.

On the experimental side, experiments at a filling factor of 2 with a hot electron distribution in one channel have demonstrated that the inelastic length is of the order of $3 \mu \mathrm{m}$ [13]. This length is of the order of the separation between the QD and the MZI, meaning that electrons are only partially relaxed. A second experimental evidence against fast relaxation is the Hong-Ou-Mandel (HOM) experiment with electrons injected one by one into edge states via quantum dots, which are excited with a periodic radio-frequency gate voltage matched to their addition energy [3]. The observed HOM dip, resulting from the collision of two electrons emerging from two separate sources, implies a coherent propagation of the injected electrons. This observation, for which electrons have been injected at energies higher than $1 \mathrm{meV}$, shows qualitatively that an important fraction of the injected electrons remains coherent, as observed in our experiment.

Furthermore, our quantitative model calculations show that the electrons reaching the MZI are only partially relaxed. We describe our system through an approach similar to Ref. [22], which considers the relaxation of an energy resolved single electron wave packet $\phi_{k_{0}}(x) \propto e^{i k_{0} x}$ injected in the outer edge channel, and takes into account the coupling of the $v=2$ edge channels [4]. This approach has proved useful for the understanding of unexpected phenomena observed on out-of-equilibrium MZIs [17], and of the observed energy transfer between edge channels $[13,23]$. The coupling to the electromagnetic environment, responsible for the electronic decoherence, is described by an electrostatic coupling to a metallic electrode, itself connected to the ground by an arbitrary impedance at an arbitrary temperature. This idealized picture is expected to be valid for electrons having a typical injection energy $\varepsilon_{0}=\hbar v_{F} k_{0} \gg \Delta \epsilon$, with $\Delta \epsilon$ their energy width. It is a criterion that is valid in our experiment, and as long as the dephasing environment can be considered as linear and in thermal equilibrium. Based on our previous experiments on phase coherence in the integer quantum Hall effect (IQHE) at $v=2[11,12]$, we assume that the electromagnetic environment of the interfering edge state consists of the co-propagating edge state, which replaces the metallic gate. Furthermore, as we probe the phase coherence of electrons injected at energies above the Fermi sea much higher than the thermal energy, we assume a zero temperature of the neighboring edge mode. The effect of its finite temperature is then simply taken into account in the finite visibility of Aharonov-Bohm (AB) interferences at zero energy. The two main outcomes of this approach to quasiparticle relaxation are the electron distribution $\delta n\left(k, k_{0}, L\right)$ and the elastic scattering probability $\mathcal{Z}\left(k_{0}, L\right)$ after a finite propagation length $L$. The visibility is then obtained from $\int \delta n\left(k, k_{0} L\right) \mathcal{Z}\left(2 k_{0} L\right) d k$.

To include the relaxation on the length $L_{\mathrm{QD}}$ separating the QD from the MZI, we computed $\delta n\left(k, k_{0} L_{\mathrm{QD}}\right)$ and the full theoretical $\mathcal{V}_{\text {Theo. }} \propto \int \delta n\left(k, k_{0} L_{\mathrm{QD}}\right) \mathcal{Z}\left(2 k L_{\mathrm{MZI}}\right) d k$, where $L_{\mathrm{MZI}}$ is the MZI arm length. The inclusion of this length shows that the electronic wave packet is partially (and not totally) relaxed before entering the MZI. The theoretical results are plotted in Fig. 4 for two different values of the coupling parameter $\theta$ measured in recent experiments [24,25]. In order to fit our data and the decrease of the visibility at low energy, we chose a drift velocity of $5 \times 10^{4} \mathrm{~ms}^{-1}$. However, the general appearance of the curves does not qualitatively change for reasonable values of the coupling strength and of the drift velocity (see SM [21]).

As expected, but in contradiction with our observations, the theory does not lead to a saturation of the visibility at high energy, but to a continuous decrease, albeit with a slope less pronounced than at low energy. This continuous decrease can be easily understood: For a short range interaction, the environment can absorb any energy; hence, the higher the injection energy, the larger is the probability for energy losses. This argument is in fact quite general and should apply regardless of the microscopic details of the model. Hence, although the other existing models [4,26-28] have not treated explicitly the decoherence of electrons injected in a narrow energy window above the Fermi level, we do not expect they would yield predictions qualitatively different from ours, as they also start from a short range Coulomb interaction between co-propagating edge channels. Last, a recent theoretical paper [29] based on short range interaction reaches the same conclusions. Our experimental observation of a saturation of the high energy visibility suggests the existence of another not yet identified energy scale in the system, or suggests the presence of an additional relaxation mechanism which remains to be identified. A somewhat similar effect has been probed at lower filling factors and much higher energies [30].

The robustness of the quantum coherence, which we have demonstrated here, increases the attraction of electron guns for quantum optics type experiments. At the same time, our results raise questions regarding our understanding of excitations in the integer quantum Hall regime. To further elucidate the relaxation in edge states, the next step in this experiment will be to implement a second 
quantum dot after the MZI, to make the spectroscopy of the injected electron after its propagation through the interferometer.
P.R. would like to thank Eugene Sukhorukov for stimulating discussions. This work has been supported by the French ANR Contract No. 11-BS04-022-01 IQHAR.
[1] Y. Ji, Y. Chung, D. Sprinzak, M. Heiblum, D. Mahalu and H. Shtrikman, Nature (London) 422, 415 (2003).

[2] G. Fève, A. Mahé, J.-M. Berroir, T. Kontos, B. Plaçais, D. C. Glattli, A. Cavanna, B. Etienne, and Y. Jin, Science 316, 1169 (2007).

[3] E. Bocquillon, V. Freulon, J.-M Berroir, P. Degiovanni, B. Plaçais, A. Cavanna, Y. Jin, and G. Fève, Science 339, 1054 (2013).

[4] I. P. Levkivskyi and E. V. Sukhorukov, Phys. Rev. B 78, 045322 (2008).

[5] C. W. J. Beenakker, C. Emary, M. Kindermann, and J. L. van Velsen, Phys. Rev. Lett. 91, 147901 (2003).

[6] P. Samuelsson, E. V. Sukhorukov, and M. Büttiker, Phys. Rev. Lett. 92, 026805 (2004).

[7] G. Haack, M. Moskalets, J. Splettstoesser, and M. Buttiker, Phys. Rev. B 84, 081303(R) (2011).

[8] G. Rosselló, F. Battista, M. Moskalets, and J. Splettstoesser, Phys. Rev. B 91, 115438(R) (2015).

[9] R. C. Ashoori, H. L. Stormer, L. N. Pfeiffer, K. W. Baldwin, and K. West, Phys. Rev. B 45, R3894 (1992).

[10] N. Kumada, H. Kamata, and T. Fujisawa, Phys. Rev. B 84, 045314 (2011).

[11] P. Roulleau, F. Portier, D. C. Glattli, P. Roche, A. Cavanna, G. Faini, U. Gennser, and D. Mailly, Phys. Rev. Lett. 100, 126802 (2008).

[12] P. Roulleau, F. Portier, P. Roche, A. Cavanna, G. Faini, U. Gennser, and D. Mailly, Phys. Rev. Lett. 101, 186803 (2008).

[13] H. le Sueur, C. Altimiras, U. Gennser, A. Cavanna, D. Mailly, and F. Pierre, Phys. Rev. Lett 105, 056803 (2010).

[14] I. Neder, M. Heiblum, Y. Levinson, D. Mahalu, and V. Umansky, Phys. Rev. Lett. 96, 016804 (2006).

[15] P. Roulleau, F. Portier, D. C. Glattli, P. Roche, A. Cavanna, G. Faini, U. Gennser, and D. Mailly, Phys. Rev. B 76, 161309(R) (2007).
[16] L. V. Litvin, H. P. Tranitz, W. Wegscheider, and C. Strunk, Phys. Rev. B 75, 033315 (2007).

[17] P.-A. Huynh, F. Portier, H. le Sueur, G. Faini, U. Gennser, D. Mailly, F. Pierre, W. Wegscheider, and P. Roche, Phys. Rev. Lett. 108, 256802 (2012).

[18] L. D. Landau, Sov. Phys. JETP 3, 920 (1957).

[19] L. D. Landau, Sov. Phys. JETP 8, 70 (1959).

[20] C. W. J. Beenakker, Phys. Rev. B 44, 1646 (1991).

[21] See Supplemental Material at http://link.aps.org/supplemental/ 10.1103/PhysRevB.93.035420 for details on the Quantum Dot tuning as well as a longer explanation of the theoretical approach.

[22] P. Degiovanni, C. Grenier, and G. Fève, Phys. Rev. B 80, 241307(R) (2009).

[23] P. Degiovanni, C. Grenier, G. Fève, C. Altimiras, H. le Sueur, and F. Pierre, Phys. Rev. B 81, 121302(R) (2010).

[24] H. Inoue, A. Grivnin, N. Ofek, I. Neder, M. Heiblum, V. Umansky, and D. Mahalu, Phys. Rev. Lett. 112, 166801 (2014).

[25] E. Bocquillon, V. Freulon, J.-M. Berroir, P. Degiovanni, B. Plaçais, A. Cavanna, Y. Jin, and G. Fève, Nat. Commun. 4, 1839 (2013).

[26] L. Chirolli, F. Taddei, R. Fazio, and V. Giovannetti, Phys. Rev. Lett. 111, 036801 (2013).

[27] M. J. Rufino, D. L. Kovrizhin, and J. T. Chalker, Phys. Rev. B 87, 045120 (2013).

[28] C. Wahl, J. Rech, T. Jonckheere, and T. Martin, Phys. Rev. Lett. 112, 046802 (2014).

[29] A. O. Slobodenisk, E. G. Idrisov, and E. V. Sukhorukov, Phys. Rev. B 93, 035421 (2016).

[30] J. D. Fletcher, P. See, H. Howe, M. Pepper, S. P. Giblin, J. P. Griffiths, G. A. C. Jones, I. Farrer, D. A. Ritchie, T. J. B. M. Janssen, and M. Kataoka, Phys. Rev. Lett. 111, 216807 (2013). 